\title{
Development of dihydrochalcone-functionalized gold nanoparticles for augmented antineoplastic activity
}

This article was published in the following Dove Press journal: International Journal of Nanomedicine

\author{
Jason N Payne ${ }^{1,2, *}$ \\ Vivek D Badwaik ${ }^{3, *}$ \\ Hitesh K Waghwani ${ }^{2, *}$ \\ Harsh V Moolani ${ }^{2}$ \\ Sarah Tockstein ${ }^{2}$ \\ David H Thompson ${ }^{3}$ \\ Rajalingam \\ Dakshinamurthy \\ 'Department of Chemistry, Austin \\ Peay State University, Clarksville, \\ TN, USA; ${ }^{2}$ Department of Chemistry, \\ Western Kentucky University, Bowling \\ Green, KY, USA; ${ }^{3}$ Department of \\ Chemistry, Multi-disciplinary Cancer \\ Research Facility, Bindley Bioscience \\ Center, West Lafayette, IN, USA \\ *These authors contributed equally \\ to this work
}

Background: Phloridzin, an antidiabetic and antineoplastic agent usually found in fruit trees, is a dihydrochalcone constituent that has a clinical/pharmaceutical significance as a sodiumglucose linked transport 2 (SGLT2) inhibitor. While the aglycone metabolite of phloridzin, phloretin, displays a reduced capacity of SGLT2 inhibition, this nutraceutical displays enhanced antineoplastic activity in comparison to phloridzin.

Purpose: The objective of this study was to develop gold nanoparticle (AuNP) mediated delivery of phloridzin and phloretin and explore their anticancer mechanism through conjugation of the dihydrochalcones and the AuNP cores.

Methods: Phloridzin and phloretin conjugated AuNPs (Phl-AuNP and Pht-AuNP) were synthesized in single-step, rapid, biofriendly processes. The synthesized AuNPs morphology was characterized via transmission electron microscopy and ultraviolet-visible spectroscopy. The presence of phloridzin or phloretin was confirmed using scanning electron microscopy-energy dispersive $\mathrm{x}$-ray spectroscopy. The percentage of organic component (phloridzin/phloretin) onto AuNPs surface was characterized using thermogravimetric analysis. Assessment of the antineoplastic potency of the dihydrochalcones conjugated AuNPs against cancerous cell lines (HeLa) was accomplished through monitoring via flow cytometry.

Results: The functionalized AuNPs were synthesized via a single-step method that relied only upon the redox potential of the conjugate itself and required no toxic chemicals. The synthesized Phl-AuNPs were found to be in the size range of $15 \pm 5 \mathrm{~nm}$, whereas the Pht-AuNP were found to be $8 \pm 3 \mathrm{~nm}$, placing both conjugated AuNPs well within the size range necessary for successful pharmaceutical applications. These assays demonstrate a significant increase in the cancerous cell toxicities as a result of the conjugation of the drugs to AuNPs, as indicated by the 17.45-fold increase in the efficacy of Pht-AuNPs over pure phloretin, and the 4.49-fold increase in efficacy of Phl-AuNP over pure phloridzin.

Conclusion: We report a simple, biofriendly process using the reducing and capping potential of the dihydrochalcones, phloridzin and phloretin, to synthesize stable AuNPs that have promising futures as potential antineoplastic agents.

Keywords: gold nanoparticles, cancer, phloretin, phloridzin

\section{Introduction}

As of 2016, approximately 1,685,210 individuals in the United States were diagnosed with some form of cancer. ${ }^{1}$ This rate has been steadily increasing over the past few decades, which has led to a frantic search for effective cancer treatments. As of 2014, 1,830 drug treatments had been identified and developed. ${ }^{2}$ This seemingly large value is inadequate and minuscule in comparison to the number of cancer diagnosed cases. 
Additionally, most of these treatments are derivatives of chemotherapy, which may induce significant side effects. The need for more effective treatment that can be used in combination with some less malignant treatments is of paramount importance to the medical community.

Dihydrochalcones have been established as potent compounds for a vast array of pharmaceutical applications such as antidiabetic, antifungal, anti-inflammatory, antimalarial, antibacterial and antineoplastic medications. ${ }^{3-7} \mathrm{~A}$ dihydrochalcone pair of particular interest in this study are phloridzin (1-[2,4-dihydroxy-6-[(2S,3R,4R,5S,6R)-3,4,5trihydroxy-6-(hydroxymethyl)tetrahydropyran-2-yl]oxyphenyl]-3-(4-hydroxyphenyl)propan-1-one) and its aglycone, phloretin (3-(4-Hydroxyphenyl)-1-(2,4,6-trihydroxyphenyl)1-propanone). Phloridzin was initially isolated for pharmaceutical usage as the first sodium-glucose linked transporter 2 (SGLT2) inhibitor in 1838. ${ }^{8,9}$ The mechanism of action for phloridzin allowed for the inhibition of glucose absorption through the SGLT2 due to a binding affinity for SGLT2 that was 3,000 times greater than that of glucose. ${ }^{9-11}$ Given this mechanism, phloridzin was suggested as an appropriate treatment for hyperglycemia; however, phloridzin never achieved widespread usage due to its poor selectivity and poor oral bioavailability in comparison to other drug options. The phloridzin's poor oral bioavailability is primarily attributed to the presence of lactase-phloridzin hydrolase in the microvillar membrane of the small intestine. ${ }^{12}$ This enzyme, intended for the hydrolysis of lactose, hydrolyses the $\beta$-glycosidic bond of phloridzin, resulting in the formation of phloretin and glucose. Phloretin demonstrates more modest antidiabetic activity, in comparison to phloridzin, due to its mechanism of action, which focuses on glucose transporter (GLUT) inhibition. ${ }^{13}$ Since phloretin's mechanism is much less specific than that of phloridzin, it is ultimately less effective for the treatment of hyperglycemia. Through comprehensive pharmaceutical experimentation, it was determined that in addition to potential pharmaceutical application of phloridzin and phloretin as an antidiabetic agent, these nutraceuticals demonstrated a significant effect as antineoplastic, antipyretic and antimalarial agents. ${ }^{9,11,14,15}$ Of particular interest are the potential applications of phloridzin and phloretin as antineoplastic agents. The efficacy of phloridzin and phloretin has been previously examined; it was determined that both compounds demonstrate a statistically significant antineoplastic action, where phloretin is more potent than phloridzin..$^{5,6,16,17} \mathrm{In}$ order to properly exploit this activity to its greatest potential, a drug delivery system must be integrated with these drugs to side-step any inherent bioavailability and selectivity issues.

Nanoscale materials bring a plethora of new possibilities to medicinal chemistry. Gold nanoparticles (AuNPs) have proven to be particularly useful in medicinal chemistry due to their chemical stability, ease of surface functionalization, and relative safety, and have been used for several years for cancer drug delivery and bioimaging. ${ }^{18-21}$ Clinicallyapproved, nano-conjugated doxorubicin have demonstrated an enhanced drug accumulation and retention in multidrug resistant $\mathrm{MCF}-7 / \mathrm{ADR}$ cancer cells. ${ }^{22-26}$ Provided with the highly hydroxylated structure of both phloridzin and phloretin, conjugation via hydroxyl group oxidation as an ester linkage to the AuNP structure is possible (Figure 1). These studies, among others, provide credible evidence that the conjugation of phloridzin and phloretin to AuNPs have the potential for augmented antineoplastic activity. ${ }^{23,26,27}$ The successful conversion of an antiquated antidiabetic treatment (phloridzin) and its biodegrade precursor (phloretin) to a successful antineoplastic treatment would invigorate and revitalize such efforts, possibly leading to a new wave of effective, cost-effective antineoplastic treatments.

\section{Materials and methods Materials}

Analytical grade phloridzin (Sigma Aldrich Co., St Louis, MO, USA), analytical grade phloretin (Sigma Aldrich<smiles>O=C(CCc1ccc(O)cc1)c1c(O)cc(O)cc1O</smiles><smiles>O=C(CCc1ccc(O)cc1)c1ccccc1OC1O[C@H](CO)[C@@H](O)[C@H](O)[C@H]1O</smiles>

Figure I Chemical structures of the two dihydrochalcones.

Note: (A) Phloretin molecular weight: $274.26 \mathrm{~g} \mathrm{~mol}^{-1}$; (B) phloridzin molecular weight: $342.30 \mathrm{~g} \mathrm{~mol}^{-1}$. 
Co.) potassium gold (III) chloride (Sigma Aldrich Co.), gold (III) chloride trihydrate (Sigma Aldrich Co.), sodium citrate dihydrate (Sigma Aldrich Co.), Dulbecco's Modified Eagle's Media (Corning Inc. Corning, NY, United States), fetal bovine serum (Life Technologies, Thermo Fisher Scientific, Waltham, MA, USA), and Sytox 7AAD (Life Technologies, Thermo Fisher Scientific), were purchased and used as required. The HeLa cell line was purchased commercially from the American Type Culture Collection (ATCC, Manassas, VA, USA). All laboratory glassware were thoroughly cleaned and rinsed followed by steam sterilization $\left(121^{\circ} \mathrm{C}, 45 \mathrm{~min}\right)$ before use. The cancerous cells were cultured by standard procedures.

\section{Synthesis of phloretin-gold nanoparticles (Pht-AuNPs)}

To synthesize the Pht-AuNPs, a primary reaction mixture was prepared of $0.365 \mathrm{M}$ phloretin in $100 \%$ ethanol. A secondary reaction mixture of autoclaved deionized water (pH: 7.2 \pm 0.2 ) was preheated in a Thermo Scientific water bath at $80^{\circ} \mathrm{C}$ for 20 minutes. Once adequate heating of the secondary mixture was achieved, an aliquot of the primary reaction mixture (phloretin in ethanol) was added to result in a $1.22 \mathrm{mM}$ phloretin solution. This reagent mixture was removed from the water bath after the addition of the ligand, at which point an aliquot of $0.132 \mathrm{M} \mathrm{KAuCl}_{4}$ was added to the solution to a final concentration of $0.353 \mathrm{mM} \mathrm{KAuCl}_{4}$. After mixing, a visible colorimetric shift from colorless to dark red was observed, indicating the formation of Pht-AuNPs. The reaction mixture was subsequently subjected to at least seven cycles of washing and centrifugation (14,000 rpm for 1 hour) with autoclaved deionized water to remove any unreacted reagents. Following the washing, the Pht-AuNP solution was pelleted, lyophilized, and stored at $4^{\circ} \mathrm{C}$ for stability. The Pht-AuNPs were reconstituted into an aqueous mixture with autoclaved deionized water and probe sonicated for most subsequent characterization and analysis.

\section{Synthesis of phloridzin-gold nanoparticles (Phl-AuNPs)}

To synthesize the Phl-AuNPs, a primary reaction mixture was prepared of $0.953 \mathrm{mM}$ phloridzin in autoclaved deionized water ( $\mathrm{pH}: 7.2 \pm 0.2$ ). This reagent mixture was preheated in a Thermo Scientific water bath at $90^{\circ} \mathrm{C}$ for 10 minutes. Once adequate heating of the reagent mixture was achieved, an aliquot of $0.132 \mathrm{M} \mathrm{KAuCl}_{4}$ was added to the solution to a final concentration of $1.50 \mathrm{mM} \mathrm{KAuCl}_{4}$. This reagent mixture was removed from the water bath after the addition of the $\mathrm{KAuCl}_{4}$. After mixing, a visible colorimetric shift from colorless to purple was observed, indicating the formation of Phl-AuNPs. The reaction mixture was subsequently subjected to at least 10 cycles of washing and centrifugation (14,000 rpm for 20 minutes) with autoclaved deionized water to remove any unreacted reagents. Following the washing, the Phl-AuNP solution was pelleted, lyophilized, and stored at $4^{\circ} \mathrm{C}$ for stability. The Phl-AuNPs were reconstituted into an aqueous mixture with autoclaved deionized water and probe-sonicated for most subsequent characterization and analysis.

\section{Characterization of phloridzin- and phloretin-coated NPs}

Characterization of the Pht-AuNP and Phl-AuNP samples via ultraviolet-visible (UV-Vis) spectroscopy was performed with a Hitachi U-3900 spectrophotometer at a resolution of $0.5 \mathrm{~nm}$. The samples were prepared creating $1 \mathrm{mg} \mathrm{mL}^{-1}$ concentrations of both AuNP samples aliquoted in quartz crystal cuvettes. This analysis was intended to observe the peaks generated by the characteristic optical properties of AuNPs known as surface plasmon resonance (SPR). To characterize the morphological properties of the AuNP, such as size and shape, the samples were examined through transmission electron microscopy (TEM) using a JEOL JEM 1400Plus electron microscope operating at a $110 \mathrm{kV}$ accelerating voltage. Sample preparation for TEM characterization involved the placement of $5 \mu \mathrm{L}$ of each sample suspension on formvar-coated, 400-mesh copper grids, which were allowed to air-dry for 1 hour. Images of the samples were acquired through the built-in AMT XR-81M-B camera, and were subsequently processed via the Capture Engine Software AMT Version 602.600.52. The diameter and average size distribution of the AuNPs were determined using a Zetasizer Nano S (Malvern Instruments, Malvern, UK) dynamic light scattering analyzer at $25^{\circ} \mathrm{C}$ with a scattering angle of $90^{\circ}$. For this analysis a sample preparation, $1 \mathrm{~mL}$ of $2 \mathrm{mg} \mathrm{mL}^{-1}$ AuNPs suspension was prepared and probe sonicated. The size was reported as an average distribution for over 40 runs for each respective sample. Presence of chalcone ligands on the surface of the AuNPs was confirmed from the surface elemental analysis using a JEOL-JSM-6510 LV scanning electron microscope (SEM) with IXRF system. For analysis, $50 \mu \mathrm{L}$ of $1 \mathrm{mg} \mathrm{mL}^{-1}$ sonicated samples of Phl-AuNP and Pht-AuNP suspensions were evenly spread on a cleaned silicon wafer and dried under vacuum at $80^{\circ} \mathrm{C}$. SEM images of substrates were obtained at $20 \mathrm{kV}$ accelerating voltage and $25 \mathrm{kX}$ magnification followed by surface elemental analysis using energy dispersive spectroscopy (EDS). The presence and percentage of phloridzin and phloretin (w/w) 
were confirmed using thermo-gravimetric analysis (TGA). Approximately $5 \mathrm{mg}$ of lyophilized AuNPs and pure phloridzin and phloretin powder was heated individually in a platinum pan over a temperature range of $25^{\circ} \mathrm{C}-850^{\circ} \mathrm{C}$ at a heating rate of $10^{\circ} \mathrm{C} \mathrm{m^{-1 }}$ in the presence of nitrogen $\left(\mathrm{N}_{2}\right)$ gas using a TA Q5000 instrument. The $\mathrm{N}_{2}$ gas was changed to air after $650^{\circ} \mathrm{C}$ to allow complete oxidation. Thermograms showing the gradual weight loss of the ligand upon heating of the Phl-AuNPs and Pht-AuNPs were plotted and compared to the pure form of the ligands. The electrostatic charge and stability of the NPs were determined using a Zetasizer Nano S (Malvern Instruments). Then $1 \mathrm{mg} \mathrm{mL}^{-1}$ AuNP suspensions were prepared by dissolving the samples in deionized water. The $\mathrm{pH}$ of the solution was maintained at a $\mathrm{pH}$ of $7.2 \pm 0.2$ throughout the analysis. The analysis was performed at $25^{\circ} \mathrm{C} \pm 0.3^{\circ} \mathrm{C}$, at a scattering angle of $90^{\circ}$, and the applied voltage was $100 \mathrm{~V}$. Results were reported as the average of 40 measurements for each sample's $\zeta$-potential determination. $^{28}$

\section{Synthesis and characterization of Citrate-AuNPs}

The Turkevich method was employed to synthesize the citrate-conjugated AuNPs. ${ }^{29}$ Briefly, $1 \mathrm{mM}$ Aurochloric acid was preheated to $100^{\circ} \mathrm{C}$ with a $1 \%$ solution of sodium citrate dihydrate. ${ }^{30}$ A colorimetric shift from colorless to dark red was indicative of the formation of Citrate-AuNPs. The reaction mixture was subsequently removed from the heating source and allowed to cool to $25^{\circ} \mathrm{C}$ before the solution was titrated to a $\mathrm{pH}$ of 7.2 \pm 0.2 . Unbound reactants were removed by washing with autoclaved nanopure water and centrifugation (15,000 rpm for 20 minutes) five times. After the final wash, Citrate-AuNPs were pelleted, lyophilized, and stored at room temperature until re-suspended in autoclaved deionized water for characterization and analysis. Citrate-AuNPs were characterized by TEM and the zeta potential measured as described above for Phl-AuNPs and Pht-AuNPs. ${ }^{28}$

\section{Evaluation of Phl-AuNPs and Pht-AuNPs for antineoplastic activity}

To assess the antineoplastic activity of the synthesized AuNPs, in comparison to their free drug forms, all samples were prepared for analysis via live/dead cell staining assays preformed through flow-cytometry in HeLa cells. Stock solutions of the samples were prepared as $4 \mathrm{mg} \mathrm{mL}^{-1}$ solutions in Dulbecco's Modified Eagle's Media (DMEM; serum free). Solvation was assured through mixing and incubation at $35^{\circ} \mathrm{C}-45^{\circ} \mathrm{C}$. Dilutions of the main stock solutions were prepared using DMEM as the solvent. The HeLa cells were cultured in DMEM supplemented with $10 \%$ fetal bovine serum at $37^{\circ} \mathrm{C}$, in $5 \% \mathrm{CO}_{2}$ and $95 \%$ relative humidity. The resultant cells were seeded in 24-well plates at cell densities of 75,000 cell/well. After 24 hours, the culture media was replaced with the previously prepared serum-free culture media containing increasing concentrations of AuNPs and pure drugs. The plates were incubated for 1 hour and 4 hours after the inoculation with the sample culture media. After the respective incubation periods for the plates, the AuNPs and pure drugs were removed via several washes with a phosphate buffer solution ( $\mathrm{pH}: 7.2 \pm 0.2)$, and fresh DMEM supplemented with 10\% fetal bovine serum was added to each well. After a further incubation period of 36 hours, the media was aspirated and the cells were washed with a phosphate buffer solution. Following the wash, the cells underwent trypsinization to remove them from their plates and were then added to fluorescence-activated cell sorting tubes. To each tube $1.0 \mu \mathrm{L}$ of Sytox 7AAD dead cell stain was added and incubated in an ice bath at $0^{\circ} \mathrm{C}-4^{\circ} \mathrm{C}$ for 15 minutes prior to fluorescence-activated cell sorting analysis. Fluorescence channel 4 (FL4) was used for the 7AAD fluorescence analysis. Cell viability percentage was calculated relative to the untreated cells which were considered as $100 \%$ viability.

\section{Results and discussion Synthesis and characterization of Pht-AuNPs and Phl-AuNPs}

The synthesis of AuNPs has been traditionally a multi-step process that relied upon the use of several chemical reagents, namely reducing agents and capping agents..$^{21,29-32}$ In general, the process of AuNP formation begins with the addition of a reducing agent to a gold salt solution, composed of gold ions $\left(\mathrm{Au}^{3+} / \mathrm{Au}^{2+}\right)$, to yield reduced neutral gold atoms. These ground state gold atoms demonstrate a propensity to aggregate in a manner that results in a varied shaped,

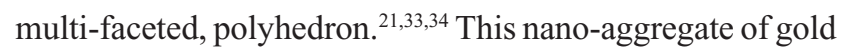
atoms would expand without limit unless a capping agent is introduced into the system to limit the size, and in certain cases, the spatial dimensions. ${ }^{29,30,32,35}$ In traditional synthesis, methodologies rely on such multi-step processes to ensure the formation of consistent and stable NPs. However, such methods require additional steps for purification due to the production of reagent by-products, which ultimately result in the process having limited scalability due to its labor- and material-intensive nature. The methodologies employed in this synthesis procedure allow for a single-pot reaction mixture that is conveniently and completely biofriendly, which 
is highly desirable given the potential medicinal application of the AuNPs produced.$^{36}$ In this synthetic methodology employed, the numerous electron rich regions of both of the chalcones (phloridzin and phloretin) serve as the reducing and, ultimately, the capping agent. The optimum concentration of the reagents, gold salt and phloridzin or phloretin, for AuNP synthesis was determined through assessment of the AuNP synthesis efficiency of a vast array of differing concentrations. Through these arrays it was determined that for the optimum synthesis of phloridzin a reaction mixture of $0.953 \mathrm{mM}$ phloridzin and $1.50 \mathrm{mM} \mathrm{KAuCl}$ in water produced the optimum yield of Phl-AuNPs. In the synthesis of Pht-AuNPs, it was determined that in order to achieve adequate solvation of the potential ligand the phloretin, a $0.365 \mathrm{M}$ phloretin solution in absolute ethanol must be prepared, prior to addition of phloretin to a $0.353 \mathrm{mM} \mathrm{KAuCl}_{4}$ aqueous media ( $\mathrm{pH}: 7.2 \pm 0.2$ ), yielding a $1.22 \mathrm{mM}$ solution of phloretin. These synthesis strategies resulted in the high yield of Phl-AuNPs and Pht-AuNPs, respectively, that had stable, mostly monodispersed, and uniform morphological characteristics.

Analysis of the morphological characteristics and particle aggregation character of the Phl-AuNPs and Pht-AuNPs was accomplished through the use of TEM analysis operating at
$110 \mathrm{kV}$. TEM micrographs of Phl-AuNPs revealed the NPs to all be spheroid, with low amounts of aggregation, and a particle core diameter of $15 \pm 5 \mathrm{~nm}$ (Figure 2A). Similar analysis of Pht-AuNPs revealed spheroid NPs that exhibited very little aggregation and a particle core diameter of $8 \pm 3 \mathrm{~nm}$ (Figure 2B). The size and approximate shape was confirmed through analysis of the AuNPs via UV-Vis spectroscopy by exploiting the principle characteristic of metallic NPs known as SPR, a phenomenon in which the AuNPs, when exposed to an oscillating electromagnetic field in the form of light, experience an induced oscillation of the conduction band electrons of the metallic nanoparticle. ${ }^{37-39}$ This oscillation of NP electrons results in a charge separation. The frequency at which the amplitude of this electron field is at its maximum is defined as the SPR and is characterized by a strong absorption of the incident light responsible for the oscillation. ${ }^{37,39,40}$ This characteristic is dependent upon the size of the NP in question therefore through the application of spectroscopy the size of the AuNPs and their rough spatial arrangement can be estimated. ${ }^{41,42}$ The UV-Vis spectroscopic analysis indicated peak absorptions ( $\lambda \max$ ) at $536 \mathrm{~nm}$ and $542 \mathrm{~nm}$ for Pht-AuNPs and Phl-AuNPs respectively (Figure 2C and D). Given the presence of ligands on the AuNP core, it can be inferred that the particles are spheroid and the size is in fact
A

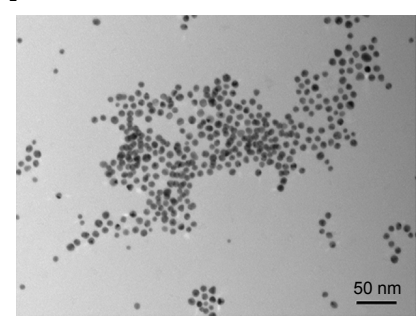

C

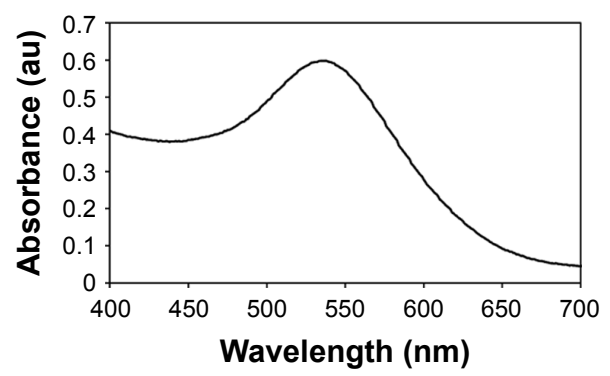

B

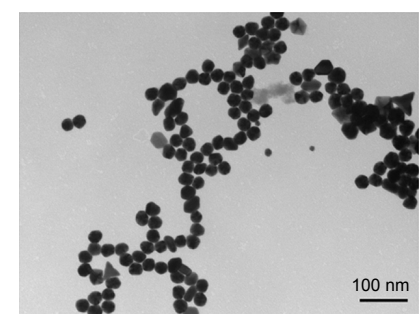

D

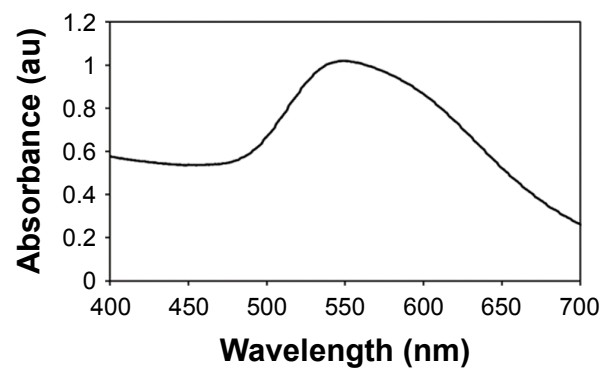

E

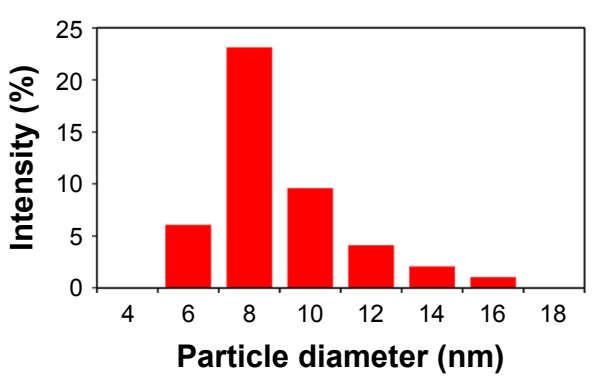

$\mathbf{F}$

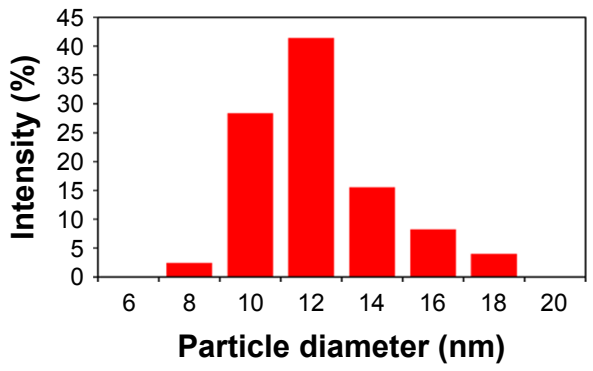

Figure 2 Illustration of the morphological and size characterization of synthesized Pht-AuNPs and Phl-AuNPs through TEM, UV-Vis, and DLS analysis. Notes: (A) TEM image showing formation of well-dispersed spherical Pht-AuNPs with a core size range of $8 \pm 3 \mathrm{~nm}(\mathrm{scale} b a r=50 \mathrm{~nm})$. (B) TEM image showing formation of well-dispersed spherical Phl-AuNPs with a core size range of $15 \pm 5 \mathrm{~nm}$ (scale bar $=100 \mathrm{~nm}$ ). (C) UV-Vis spectrogram of Pht-AuNPs showing a SPR event occurring at $536 \mathrm{~nm}$ correlating to spheroid-shaped NPs. (D) UV-Vis spectrogram of Phl-AuNPs showing a SPR event occurring at $542 \mathrm{~nm}$ correlating to spheroid-shaped NPs. (E) Pht-AuNP DLS analysis shows the average hydrodynamic diameter as $8 \pm 2 \mathrm{~nm}$. (F) Phl-AuNP DLS analysis depicts the average hydrodynamic diameter as $12 \pm 2 \mathrm{~nm}$.

Abbreviations: Pht-AuNPs, phloretin-conjugated gold nanoparticles; Phl-AuNPs, phloridzin-conjugated gold nanoparticles; TEM, transmission electron microscopy; UV-Vis, ultraviolet-visible spectroscopy; DLS, dynamic light scattering; SPR, surface plasmon resonance. 
in agreement with the data collected for TEM analysis. ${ }^{37,43}$ Through the application of DLS analysis, it was possible to determine the hydrodynamic radii distribution of the AuNPs, which, unlike the TEM core diameter determination, is a measure of the particle diameter including the conjugated ligands. ${ }^{44}$ It was determined that the hydrodynamic radii were $8 \pm 2 \mathrm{~nm}$ and $12 \pm 2 \mathrm{~nm}$ for Pht-AuNPs and Phl-AuNPs respectively (Figure $2 \mathrm{E}$ and $\mathrm{F}$ ).

In order to confirm the conjugation of the ligands (phloretin and phloridzin) to the NP, it was necessary to perform an elemental composition analysis. This characterization was achieved through scanning electron microscopy-energy dispersive x-ray spectroscopy (SEMEDS), an analytical technique that allows for the presence of carbon and oxygen to be confirmed. In this analysis the presence of both elements is confirmation of the presence of an organic ligand, specifically the drugs of interest, as the ligands are the only source of either element in the synthesis of each AuNPs. Concerning both the Pht-AuNP and Phl-AuNP analysis, it was confirmed that an organic molecule was indeed present, and given the method of sample preparation for both AuNPs that organic component must correspond to the drug being conjugated to the AuNP (Figure 3A and B). ${ }^{36}$ Quantitative analysis of the EDS spectrum produced indicated that gold accounted for $78.66 \%$ and $95.01 \%$ of the elemental composition of Pht-AuNPs and Phl-AuNPs respectively, while carbon accounted for $15.27 \%$ and $4.27 \%$ of the elemental composition of Pht-AuNPs and Phl-AuNPs respectively. Additionally, the oxidation state of the gold in the sample can be determined through EDS analysis depending on the shift of gold peak on the spectrogram. A position of approximately $2.120 \mathrm{keV}$ corresponds to reduced gold (Au0) whereas peaks of approximately 9.712 $\mathrm{keV}$ corresponds to oxidized gold species $\left(\mathrm{Au}^{2+} / \mathrm{Au}^{3+}\right){ }^{45,46}$ The spectrum indicated the strong presence of gold in the reduced state, a state that is indicative of the presence of gold in a NP formation. For a quantitative elemental composition mass analysis, thermogravimetric analysis (TGA)
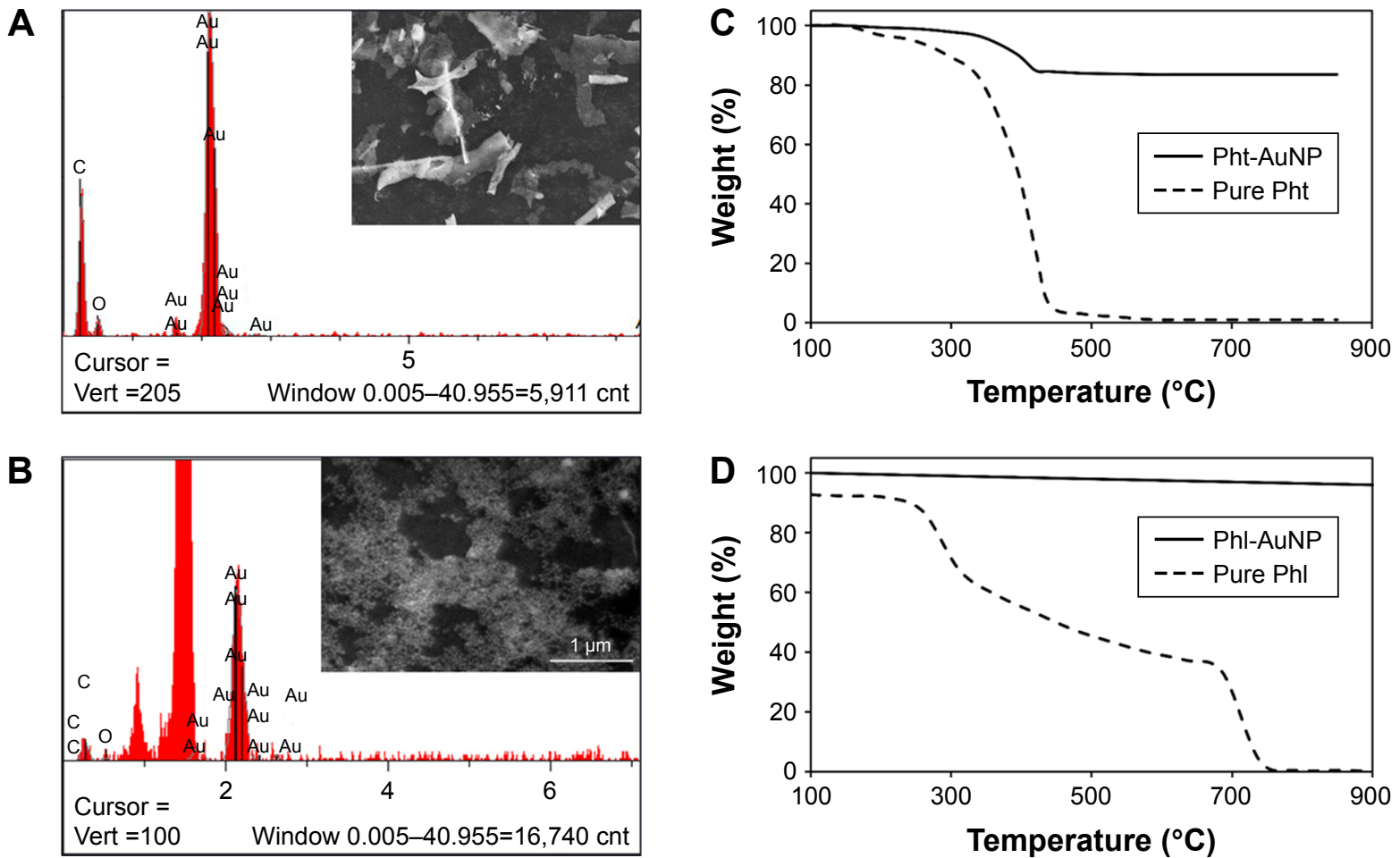

Figure 3 Characterization of Pht-AuNPs and Phl-AuNPs.

Notes: (A) EDS spectra of Pht-AuNPs showing the presence of an elemental peak for carbon (C) and gold (Au) at $0.2 \mathrm{keV}$ and $2.1 \mathrm{keV}$ respectively. Figure in the inset shows SEM image of spin coated sample of Pht-AuNPs on silicon chip obtained at an accelerating voltage of $20 \mathrm{keV}$ with a magnification of 5 kX. (B) EDS spectra of Pht-AuNPs showing the presence of an elemental peak for $\mathrm{C}$ and $\mathrm{Au}$ at $0.2 \mathrm{keV}$ and $2.1 \mathrm{keV}$ respectively. Figure in the inset shows SEM image of spin coated sample of Pht-AuNPs on silicon chip obtained at an accelerating voltage of $20 \mathrm{keV}$ with a magnification of $5 \mathrm{kX}$. (C) A comparison of TGA showing loss of organic material for Pht-AuNP ( - ) and phloretin (- - ) respectively. The samples were heated from room temperature to $650^{\circ} \mathrm{C}$ at a rate of $10^{\circ} \mathrm{C}$ min ${ }^{-1}$ under nitrogen flow followed by heating to $850^{\circ} \mathrm{C}$ under air. (D) A comparison of TGA showing loss of organic material for Phl-AuNP (-) and phloridzin (- - -) respectively. The samples were heated from room temperature to $650^{\circ} \mathrm{C}$ at a rate of $10^{\circ} \mathrm{C} \mathrm{min}^{-1}$ under nitrogen flow followed by heating to $850^{\circ} \mathrm{C}$ under air.

Abbreviations: EDS, energy dispersive spectroscopy; TGA, thermo-gravimetric analysis; Pht-AuNPs, phloretin-conjugated gold nanoparticles; Phl-AuNPs, phloridzinconjugated gold nanoparticles; SEM, scanning electron microscopy; Pht, phloretin. 
was employed. Using this method the mass percentage composition of the organic percentage can be determined from lyophilized, powder samples ensuring that the only source of organic materials is the proposed ligands. It was determined through this analysis that the mass percentage composition of Pht-AuNPs was $16.2 \%$ organic whereas the Phl-AuNPs were $4.0 \%$ organic (Figure $3 \mathrm{C}$ and D). The differing mass percentages between Pht-AuNPs and PhlAuNPs in both EDS and TGA can be accounted for due to the size of each respective ligand. Phloretin is the smaller of the two ligands as it is the aglycone of phloridzin, as such it is to be expected that more of the smaller phloretin ligand can be conjugated to each AuNP in comparison to phloridzin. Zeta potential $(\zeta)$ analysis determined the electrokinetic potential of the AuNP samples, allowing for inference to be made regarding the stability of the AuNPs in a suspension. ${ }^{47,48}$ It was determined that the zeta potential for Pht-AuNPs was $-31.7 \mathrm{mV}$ (Figure 4A) whereas the potential for Phl-AuNPs was $-38.2 \mathrm{mV}$ (Figure 4B). In the case of both Pht-AuNP and Phl-AuNP, from their zeta potentials one can conclude that they demonstrate moderate stability as the magnitude of the experimentally determined $\zeta$ is greater than $30 \mathrm{mV}$.

\section{Evaluation of Phl-AuNPs and Pht-AuNPs for antineoplastic activity}

The antineoplastic potentials of the synthesized Pht-AuNPs and Phl-AuNPs were evaluated via live/dead cell fluorescence staining assays analyzed through flow-cytometry. To perform this analysis, HeLa cells were selected to act as the model cancerous cell line. Citrate AuNPs were used as a control to evaluate the effect of the AuNP, while pure phloretin and phloridzin were used as controls to evaluate

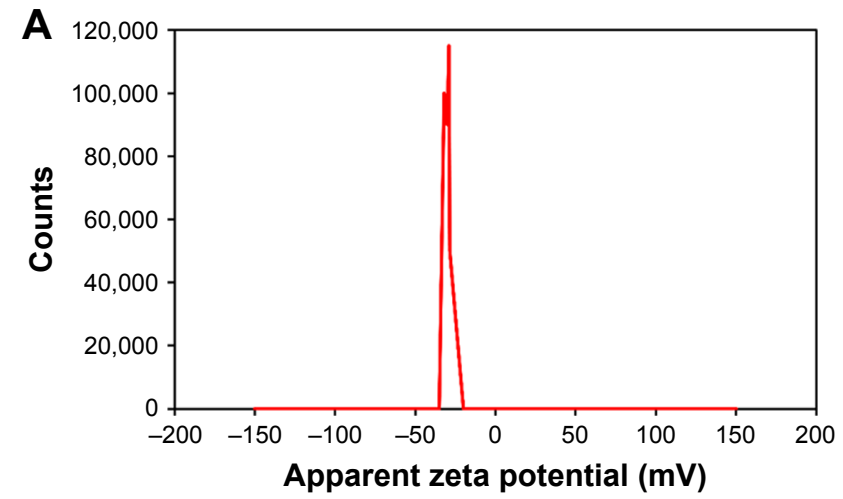

any augmented antineoplastic activity resulting from the conjugation of the drugs to the AuNP core. The efficacy of the samples was determined by the cell viability of the HeLa cells in comparison to non-inoculated HeLa cells. It was determined that inoculation times greater than 1 hour yield no statistically different cell inhibition percentages; therefore, all values represented are 1-hour post inoculation. Additionally, it was discovered that all concentrations of the samples display increasing cancerous cell cytotoxicity to a concentration of $4 \mathrm{mg} / \mathrm{mL}$. Above $4 \mathrm{mg} / \mathrm{mL}$, no significant increase in anticancer activity was observed The flowcytometry assays revealed that the pure forms of the drug ligands, phloretin and phloridzin, resulted in a HeLa cell viability of $95.000 \%$ and $88.125 \%$ respectively (Figure 5A). The assays that evaluated the synthesized AuNP conjugates, Pht-AuNPs and Phl-AuNPs, displayed a cell viability of $12.750 \%$ and $44.625 \%$ respectively (Figure $5 B$ ). The efficacy of pure AuNPs was evaluated to determine if the augmented cytotoxicity was due to a synergistic effect of the conjugation or was purely due to the presence of the AuNPs. To determine the augmented synergistic potential of AuNPs, citrate-AuNPs of comparable size to the PhtAuNPs and Phl-AuNPs $(20 \pm 5 \mathrm{~nm})$ were synthesized. The surface $\zeta$ potential of the citrate-AuNPs was determined to be $-33.5 \mathrm{mV}$. It was found, through analysis of citrateAuNPs, that cell viability remained at $85.000 \%$ indicating the increase in toxicity was a result of a synergistic activity (Figure 5C). These assays demonstrate a significant increase in the cancerous cell toxicities as a result of the conjugation of the drugs to AuNPs, as indicated by the 17.45-fold increase in the efficacy of Pht-AuNPs over pure phloretin, and the 4.49-fold increase in efficacy of Phl-AuNPs over pure phloridzin.

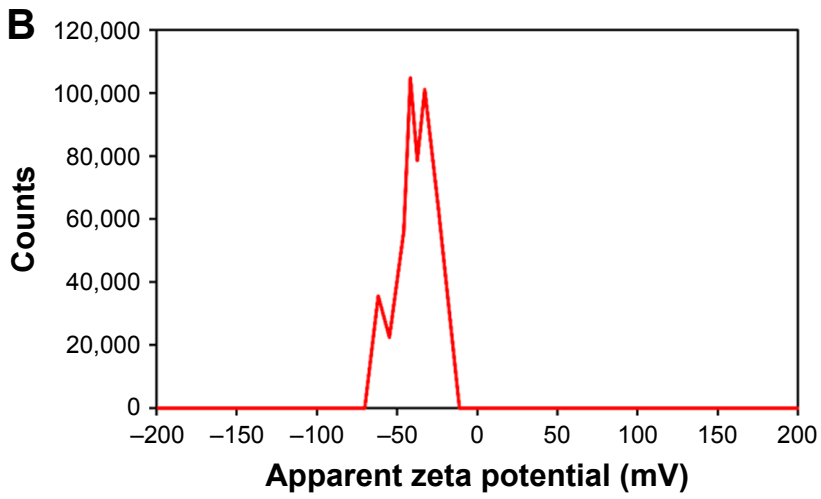

Figure 4 Surface zeta potential analysis.

Notes: (A) Surface zeta potential analysis of Pht-AuNPs determined to be $-31.7 \mathrm{mV}$, and (B) of Phl-AuNPs determined to be $-38.2 \mathrm{mV}$. The magnitude of the zeta potential greater than $30 \mathrm{mV}$ is indicative of a moderately stable AuNP suspension.

Abbreviations: Pht-AuNPs, phloretin-conjugated gold nanoparticles; Phl-AuNPs, phloridzin-conjugated gold nanoparticles; AuNP, gold nanoparticle. 

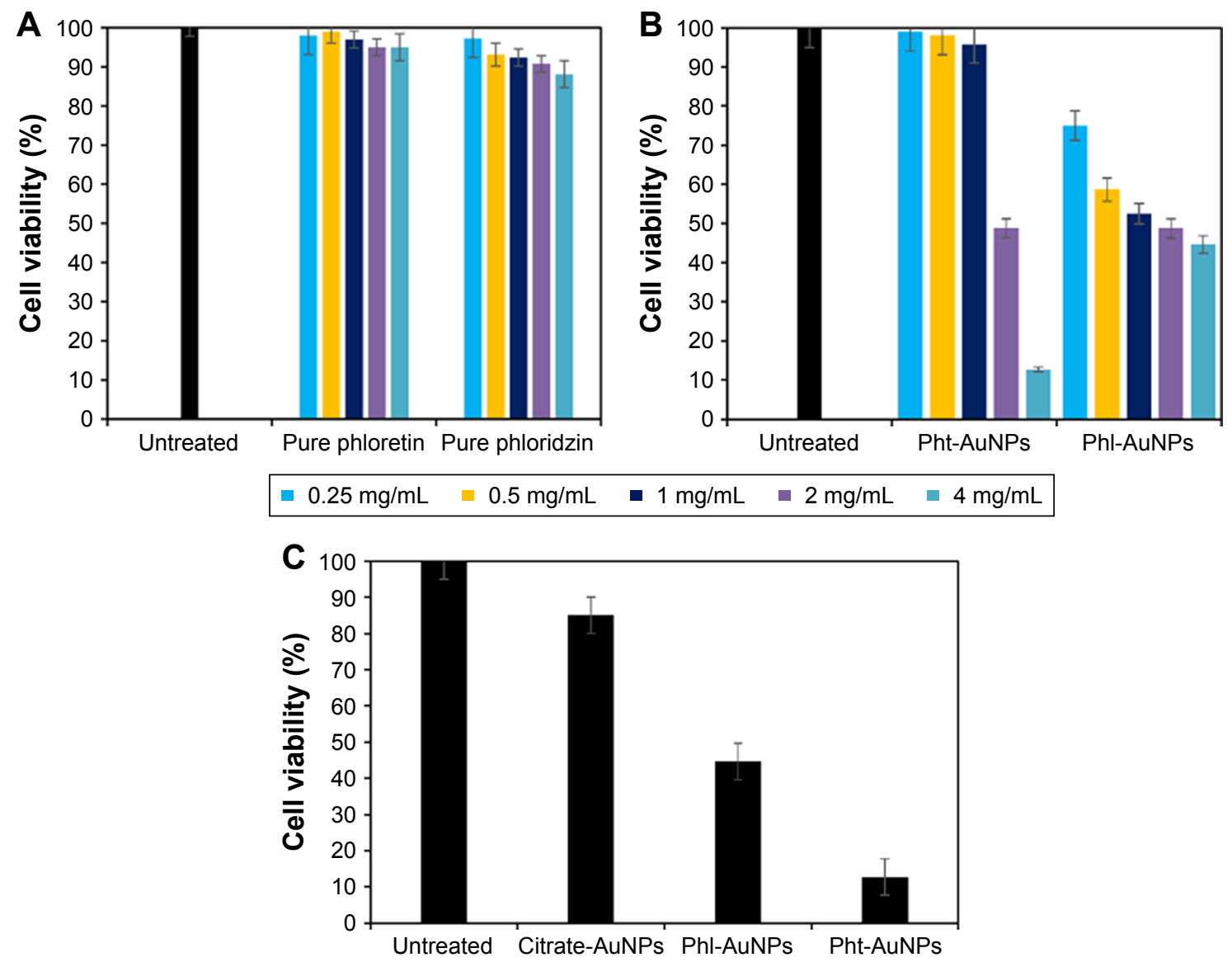

Figure 5 HeLa live/dead cell fluorescence staining analysis via flow-cytometry.

Notes: (A) Comparison of the pure drug forms of phloretin and phloridzin to non-inoculated cells. (B) Comparison of the AuNP functionalized forms of the drugs to noninoculated cells. (C) Comparison of the functionalized AuNP to pure AuNP to determine the synergistic activity of the Pht-AuNPs and Phl-AuNPs.

Abbreviations: AuNP, gold nanoparticle; Pht-AuNP, phloretin-conjugated gold nanoparticles; Phl-AuNPs, phloridzin-conjugated gold nanoparticles.

\section{Conclusion}

These results concerning the functionalization of the dihydrochalcones, phloretin and phloridzin, to AuNPs in an effort to augment the existing antineoplastic activity of each pharmaceutical agent reveal a potential treatment application. The functionalized AuNPs were synthesized via a single-step method that relied only upon the redox potential of the conjugate itself and required no toxic chemicals. The synthesized Phl-AuNPs were found to be in the size range of $15 \pm 5 \mathrm{~nm}$, whereas the Pht-AuNPs were found to be $8 \pm 3 \mathrm{~nm}$, placing both AuNP conjugates well within the size range necessary for successful pharmaceutical applications. Significant multi-fold increases in the antineoplastic activity of both phloretin and phloridzin were observed upon the functionalization of the pharmaceutical agents to AuNPs in comparison to their pure drug forms. Evaluation of the antineoplastic of non-functionalized AuNP in conjunction with this data revealed that the augmented antineoplastic activity was in fact a result of a synergistic effect between the AuNP and each of the respective drugs.
In conclusion we report a simple, bio-friendly process using the reducing and capping potential of the dihydrochalcones, phloridzin and phloretin, to synthesize stable AuNPs that have promising futures as potential antineoplastic agents.

\section{Acknowledgments}

We are grateful to Dr John Andersland for TEM analysis. Our special thanks to members of Dr Dakshinamurthy's lab, including Monic Shah and Tulsi Modi, for their assistance in the initial stages of this project and support.

The project was supported in part by American Chemical Society PRF (\#57010-UR10), Department of Chemistry at Austin Peay State University. This project is also supported by Department of Chemistry, Advanced Materials institute, Biotechnology Center, Ogden College of Science and Engineering, WKU office of Research RCAP grants, WKU Graduate School Research Fellowship, WKU FacultyUndergraduate Student Engagement (FUSE) grants, and KY NSF EPSCoR Grant (0814199). 


\section{Author contributions}

All authors contributed toward data analysis, drafting and critically revising the paper, gave final approval of the version to be published, and agree to be accountable for all aspects of the work.

\section{Disclosure}

The authors report no conflicts of interest in this work.

\section{References}

1. National Cancer Institute. NIH National Cancer Institute Cancer Statistics; 2016. Previously available from: https://seer.cancer.gov/data/2. https://seer.cancer.gov/seertools/seerrx/. Accessed June 1, 2017.

2. National Cancer Institute. SEER*Rx Interactive Antineoplastic Drugs Database; 2014.

3. Lesse WI. Ueber Diabetes Bei Frauen. Monatsschrift Für Geburtshilfe Gynäkol. 1902;15(4):680-693.

4. Norton L, DeFronzo RA, Abdul-Ghani MA. Sodium-glucose cotransporter 2 inhibition - a novel strategy for glucose control in type 2 diabetes. Eur Endocrinol. 2010;6(1):42-47.

5. Nelson JA, Falk RE. The efficacy of phloridzin and phloretin on tumor cell growth. Anticancer Res. 1993;13(6A):2287-2292.

6. Nelson JA, Falk RE. Phloridzin and phloretin inhibition of 2-deoxy-dglucose uptake by tumor cells in vitro and in vivo. Anticancer Res. 1993;13(6A):2293-2299.

7. Nakamura Y, Watanabe S, Miyake N, Kohno H, Osawa T. Dihydrochalcones: evaluation as novel radical scavenging antioxidants. $J$ Agric Food Chem. 2003;51(11):3309-3312.

8. Stas JS, Ueber das Phloridzin. J Prakt Chem. 1839;17(1):273-298.

9. Ehrenkranz JRL, Lewis NG, Kahn CR, Roth J. Phlorizin: a review. Diabetes Metab Res Rev. 2005;21(1):31-38.

10. Vick H, Diedrich DF, Baumann K. Reevaluation of renal tubular glucose transport inhibition by phlorizin analogs. Am J Physiol. 1973;224(3): 552-557.

11. Biegeleisen H. Phlorizin analogues and their use. Patent US3523937 A, August 11, 1970. Washington, DC: US Patent and Trademark Office.

12. Wüthrich M, Sterchi EE. Human lactase-phlorizin hydrolase expressed in COS-1 cells is proteolytically processed by the lysosomal pathway. FEBS Lett. 1997;405(3):321-327.

13. Lefevre PG. Sugar transport in the red blood cell: structure-activity relationships in substrates and antagonists. Pharmacol Rev. 1961;13:39-70.

14. Petersen C. Analyse des phloridzins. Ann Acad Sci Francaise. 1835; $15: 178$.

15. de Koninck L. Observations sur les proprietes febrifuges de las phloridzine. Bull Soc Sci Med. 1836;1:75-110.

16. Wang L, Li Z-W, Zhang W, et al. Synthesis, crystal structure, and biological evaluation of a series of phloretin derivatives. Molecules. 2014;19(10):16447-16457.

17. Crespy V, Aprikian O, Morand C, et al. Bioavailability of phloretin and phloridzin in rats. J Nutr. 2001;131(12):3227-3230.

18. You J-O, Guo P, Auguste DT. A drug-delivery vehicle combining the targeting and thermal ablation of HER $2+$ breast-cancer cells with triggered drug release. Angew Chem Int Ed Engl. 2013;52(15):4141-4146.

19. Zhou Y, Kong Y, Kundu S, Cirillo JD, Liang H. Antibacterial activities of gold and silver nanoparticles against Escherichia coli and bacillus Calmette-Guérin. J Nanobiotechnol. 2012;10(1):19.

20. Seil JT, Webster TJ. Antimicrobial applications of nanotechnology: methods and literature. Int J Nanomed. 2012;7:2767-2781.

21. Shah M, Badwaik VD, Dakshinamurthy R. Biological applications of gold nanoparticles. J Nanosci Nanotechnol. 2014;14(1):344-362.

22. Zhang L, Gu F, Chan J, Wang A, Langer R, Farokhzad O. Nanoparticles in medicine: therapeutic applications and developments. Clin Pharmacol Ther. 2008;83(5):761-769.
23. Aryal S, Grailer JJ, Pilla S, Steeber DA, Gong S. Doxorubicin conjugated gold nanoparticles as water-soluble and $\mathrm{pH}$-responsive anticancer drug nanocarriers. J Mater Chem. 2009;19(42):7879-7884.

24. Gu YJ, Cheng J, Man CW, Wong WT, Cheng SH. Gold-doxorubicin nanoconjugates for overcoming multidrug resistance. Nanomed Nanotechnol Biol Med. 2012;8(2):204-211.

25. Prabaharan M, Grailer JJ, Pilla S, Steeber DA, Gong S. Gold nanoparticles with a monolayer of doxorubicin-conjugated amphiphilic block copolymer for tumor-targeted drug delivery. Biomaterials. 2009; 30(30):6065-6075.

26. Wang F, Wang YC, Dou S, Xiong MH, Sun TM, Wang J. Doxorubicintethered responsive gold nanoparticles facilitate intracellular drug delivery for overcoming multidrug resistance in cancer cells. ACS Nano. 2011;5(5):3679-3692.

27. Brown SD, Nativo P, Smith JA, et al. Gold nanoparticles for the improved anticancer drug delivery of the active component of oxaliplatin. J Am Chem Soc. 2010;132(13):4678-4684.

28. Nanotechnology Characterization Laboratory. Measuring Zeta Potential of Nanoparticles, NCL Method PCC-2. Frederick, MD: National Cancer Institute-Frederick; 2009.

29. Turkevich J, Stevenson PC, Hillier J. A study of the nucleation and growth processes in the synthesis of colloidal gold. Discuss Faraday Soc. 1951;11:55.

30. Kimling J, Maier M, Okenve B, Kotaidis V, Ballot H, Plech A. Turkevich method for gold nanoparticle synthesis revisited. J Phys Chem B. 2006;110(32):15700-15707.

31. Brust M, Walker M, Bethell D, Schiffrin DJ, Whyman R. Synthesis of thiol-derivatised gold nanoparticles in a two-phase liquid-liquid system. J Chem Soc Chem Commun. 1994;(7):801-802.

32. Hutchings GJ, Brust M, Schmidbaur H. Gold - an introductory perspective. Chem Soc Rev. 2008;37(9):1759-1765.

33. Torchilin VP, editor. Handbook of Nanobiomedical Research: Fundamentals, Applications, and Recent Developments. Frontiers in Nanobiomedical Research. Hackensack, NJ: World Scientific; 2014.

34. Perala SRK, Kumar S. On the mechanism of metal nanoparticle synthesis in the Brust-Schiffrin method. Langmuir. 2013;29(31): 9863-9873.

35. Navarro JRG, Lerouge F, Cepraga C, et al. Nanocarriers with ultrahigh chromophore loading for fluorescence bio-imaging and photodynamic therapy. Biomaterials. 2013;34(33):8344-8351.

36. Dakshinamurthy R, Sahi S. Monodisperse gold nanoparticles and facile, environmentally favorable process for their manufacture. Patent US8257670 B1, September 4, 2012. Washington, DC: US Patent and Trademark Office.

37. Huang X, El-Sayed MA. Gold nanoparticles: optical properties and implementations in cancer diagnosis and photothermal therapy. $J A d v$ Res. 2010;1(1):13-28.

38. Mie G. Contributions to the optics of turbid media, particularly of colloidal metal solutions. Ann Phys. 1908;25(3):377-445.

39. Kreibig U, Vollmer M. Optical Properties of Metal Clusters. Springer Series in Materials Science, Vol. 25. Berlin, Heidelberg: Springer; 1995.

40. Papavassiliou GC. Optical properties of small inorganic and organic metal particles. Prog Solid State Chem. 1979;12(3-4):185-271.

41. Jain PK, Lee KS, El-Sayed IH, El-Sayed MA. Calculated absorption and scattering properties of gold nanoparticles of different size, shape, and composition: applications in biological imaging and biomedicine. J Phys Chem B. 2006;110(14):7238-7248.

42. Huang X, Jain PK, El-Sayed IH, El-Sayed MA. Gold nanoparticles: interesting optical properties and recent applications in cancer diagnostics and therapy. Nanomedicine. 2007;2(5):681-693.

43. Ali ME, Hashim U, Mustafa S, Che Man YB, Islam KN. Gold nanoparticle sensor for the visual detection of pork adulteration in meatball formulation. J Nanomater. 2012;2012:ID103607.

44. Khlebtsov BN, Khlebtsov NG. On the measurement of gold nanoparticle sizes by the dynamic light scattering method. Colloid J. 2011;73(1): $118-127$. 
45. Grieken RE van. Handbook of X-Ray Spectrometry. New York: Marcel Dekker; 2002.

46. Badwaik VD, Bartonojo JJ, Evans JW, Sahi SV, Willis CB, Dakshinamurthy R. Single-step biofriendly synthesis of surface modifiable, near-spherical gold nanoparticles for applications in biological detection and catalysis. Langmuir. 2011;27(9):5549-5554.
47. Greenwood R. Review of the measurement of zeta potentials in concentrated aqueous suspensions using electroacoustics. Adv Colloid Interface Sci. 2003;106:55-81.

48. O'Brien RW, Midmore BR, Lamb A, Hunter RJ. Electroacoustic studies of moderately concentrated colloidal suspensions. Faraday Discuss Chem Soc. 1990;90:301-312.

\section{Publish your work in this journal}

The International Journal of Nanomedicine is an international, peerreviewed journal focusing on the application of nanotechnology in diagnostics, therapeutics, and drug delivery systems throughout the biomedical field. This journal is indexed on PubMed Central, MedLine, CAS, SciSearch ${ }^{\circledR}$, Current Contents ${ }^{\circledR} /$ Clinical Medicine,
Journal Citation Reports/Science Edition, EMBase, Scopus and the Elsevier Bibliographic databases. The manuscript management system is completely online and includes a very quick and fair peer-review system, which is all easy to use. Visit http://www.dovepress.com/ testimonials.php to read real quotes from published authors.

Submit your manuscript here: http://www.dovepress.com/international-journal-of-nanomedicine-journal 